Thorax, 1979, 34, 547-549

\title{
Complete heart block in an adult with corrected transposition of the great arteries treated with permanent pacemaker
}

\author{
S AMIKAM, J LEMER, Y KISHON, E RISS, AND H N NEUFELD \\ From the Departments of Cardiology and Cardiac Surgery, Rambam Medical Centre, \\ Technion Faculty of Medicine, Haifa, and the Heart Institute, Haim Sheba Medical Centre, \\ Tel-Hashomer, Sackler Medical School, Tel-Aviv University, Israel
}

\begin{abstract}
A 53-year-old patient with corrected transposition of the great arteries developed complete heart block with fainting episodes. After temporary pacing through the endocardium of the venous (anatomically left) ventricle, a permanent epicardial pacemaker was implanted. This case shows the progressive nature of the atrioventricular conduction disturbances, which are very common in association with this congenital cardiac anomaly.
\end{abstract}

Congenital corrected transposition of the great arteries (CTGA) is usually accompanied by additional cardiac malformations but may occur without associated defects (Rotem and Hultgren, 1965). Atrioventricular conduction disturbances are found in almost half of the patients (Schiebler et al, 1961; Friedberg and Nadas, 1970), the commonest being a prolonged PR interval. When complete heart block is present, the QRS complex is narrow, the ventricular rate is usually greater than $50 / \mathrm{min}$, and rarely dominates the clinical picture, which is determined by the additional anatomical abnormalities (Shem-Tov et al, 1971). The few reported cases of CTGA in whom complete heart block necessitated pacemaker implantation were in children who developed this conduction disturbance in infancy, early childhood, or adolescence (Friedberg and Nadas, 1970) and only in one instance in an adult patient (Berman and Adicoff, 1969).

We describe a middle-aged patient in whom CTGA was first diagnosed at the age of 49 and who at the age of 53 developed complete atrioventricular block that required pacemaker implantation. This is the second reported adult case in whom a permanent pacemaker was implanted to treat complete heart block in the presence of CTGA.

\section{Case report}

A 53-year-old clerk was transferred to the depart- ment of cardiology at the Rambam Medical Centre with a three-day history of pronounced weakness, dyspnoea on minimal effort, and two episodes of Stokes-Adams attacks. During these episodes a slow heart rate $(30 / \mathrm{min})$ was recorded, and the electrocardiogram showed complete atrioventricular block.

In 1970 , at the age of 46 , he had begun to suffer from recurrent episodes of left heart failure and was treated with digitalis and diuretics. As he did not fully respond to this treatment he was admitted in 1973 to the heart institute of the Haim Sheba Medical Centre, where right and left heart catheterisations were performed, and the diagnosis of CTGA was established. In addition, a moderate degree of aortic regurgitation and slight left atrioventricular valve regurgitation were shown. The electrocardiogram in 1973 showed sinus rhythm with a PR interval of 0.36 seconds. The patient was discharged home, remained well, and continued to work.

At the present admission he was dyspnoeic and orthopnoeic with an irregular pulse of $54 / \mathrm{min}$ and a blood-pressure of $120 / 70 \mathrm{mmHg}$. There was no jugular venous congestion. Fine crepitations were heard at both lung bases. The apex beat was not palpable, but he had a left parasternal heave. A single and accentuated second heart sound, a $3 / 6$ ejection systolic murmur, and a $2 / 6$ early diastolic murmur were heard in the second interspace at the left sternal margin. The liver was enlarged $4 \mathrm{~cm}$ below the right costal margin. The 
spleen was not palpable. There was no ankle oedema.

Results of routine laboratory examinations were normal. A chest radiograph showed an enlarged heart with a narrow pedicle. There was severe pulmonary venous congestion, with bilateral pleural effusion. The right diaphragm was raised. The electrocardiogram showed complete atrioventricular block with occasional sinus conducted complexes. A supraventricular escape rhythm at a rate of $55 / \mathrm{min}$ with a QRS complex width of $0 \cdot 11 \mathrm{~s}$ and an axis of $+80^{\circ}$ was present.

Because of the history of recurrent StokesAdams attacks and the electrocardiographic findings of complete $\mathrm{AV}$ block, a temporary pacemaker was inserted through the left cubital vein. The electrode was introduced easily into the venous (anatomical left) ventricle and was manipulated into the pulmonary artery that was median in position (see figure). On a lateral view, the electrode within the pulmonary artery took a posterior position to the ascending aortic shadow, confirming the diagnosis of CTGA. The electrode was then withdrawn into the venous ventricle, and a satisfactory stimulation threshold was achieved.

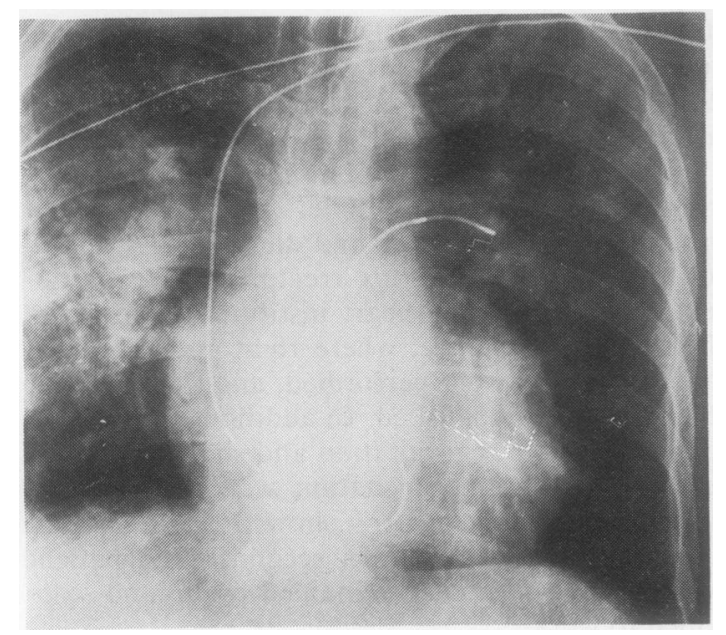

Chest radiograph obtained during insertion of temporary pacemaker. Electrode was manipulated into pulmonary artery showing its median position, which is diagnostic for corrected transposition of great arteries.

After several days of observation, when we were convinced that the complete atrioventricular block was not a result of medication or acute infarction, we decided to implant a permanent pacemaker. Because of the presence of CTGA and the uncertainty of the anatomical left ventricle devoid of trabeculations providing long-term lodgment of the endocardial electrode, an epicardial route of implantation was chosen. Under general anaesthesia, a left subcostal approach was used to implant two sutureless screw-in epicardial electrodes into the inferior surface of the heart. A demand pulse-generator (Medtronic model No 5972) was implanted.

The patient made an uneventful recovery and was discharged home ten days later. There were no further episodes of fainting during the subsequent year.

\section{Discussion}

Although corrected transposition of the great arteries is not one of the common congenital cardiac abnormalities, series of 30,33 , and 60 cases have been collected and reviewed (Schiebler et al, 1961; Friedberg and Nadas, 1970; Shem-Tov et al, $\vec{\theta}$ 1971). Atrioventricular conduction defects, vary- ب़ ing from first-degree AV block to complete heart block, were often noted in association with this anomaly, and are said to occur in one-third (Friedberg and Nadas, 1970) to two-thirds (Schiebler et $a l, 1961)$ of the cases.

It has been suggested that the $\mathrm{AV}$ conduction defects in CTGA are related to the congenitally malpositioned conduction system with an elongated AV bundle, which has to take a much longer route to reach the ventricles (Lev et al, 1963). It is more difficult, however, to explain the progressive nature of these conduction disturbances $\underset{x}{\stackrel{\nu}{\nu}}$ that has been noted in some patients with CTGA, including our patient. When CTGA was first diag- 3 . nosed at the age of 49 , the AV conduction dis- $\delta$ turbance in our patient was a prolonged PR interval. Four years later, at the age of 53, progression 0 to intermittent complete AV block was recorded and was associated with symptoms requiring pacemaker implantation. A similar progression of the AV conduction disturbances was also observed $\sigma$ by Friedberg and Nadas (1970) in five of their $N$ 60 patients with CTGA. In one, 18 years elapsed $\underset{\mathrm{N}}{\mathrm{N}}$ between normal AV conduction and the complete 0 heart block. This progression of the AV conduction disturbances over the years in patients $\frac{D}{D}$ with CTGA raises the question whether the $\mathrm{AV} \stackrel{\oplus}{?}$ block should be categorised as congenital or 0 acquired. Anderson et al (1974) found in four of their five cases who had complete heart block a $\stackrel{\mathbb{\Phi}}{\Omega}$ disruption of the anteriorly situated AV bundle $\overrightarrow{\mathbb{D}}$ by fibrous tissue. Extensive fibrosis was also ob- $\frac{\varrho}{0}$ served in the region where the anterior bundle made contact with the anterior node. They sug- 
gested that because the AV bundle in CTGA is situated anteriorly rather than posteriorly and is directly beneath the pulmonary valve in the position of mitral-pulmonary continuity, it is therefore subjected to considerable excursions during closure of the mitral valve. Possibly the fibrosis is related to this position as well as to the excessive length of the bundle. Whether this hypothesis is accepted or not, it seems that the AV conduction defect in CTGA is caused by the combination of a congenitally elongated AV bundle and acquired changes that develop throughout the years and are related to the malpositioned conducting tissue. This indicates that patients with CTGA should be followed closely, not only because of their haemodynamic state but also in view of the progressive nature of the AV conduction disturbances in this anomaly. It should be emphasised that the clinical picture of Stokes-Adams attacks, which appeared in our patient, is an unusual accompaniment of CTGA. Because of the proximal site of the conduction defect in this congenital anomaly, the escape pacemaker focus is supraventricular and therefore stable. It is the consequences of a chronic low cardiac output that dominate the clinical picture when progression to complete atrioventricular block occurs. In our patient the reason for permanent pacing was to treat low cardiac output and to prevent further StokesAdams attacks.

\section{References}

Anderson, R H, Becker, A E, Arnold, R, and Wilkinson, J L (1974). The conducting tissues in congenitally corrected transposition. Circulation, 50, 911-923.

Berman, D A, and Adicoff, A (1969). Corrected transposition of the great arteries causing complete heart block in an adult. American Journal of Cardiology, 24, 125-129.

Friedberg, D Z, and Nadas, A S (1970). Clinical profile of patients with congenital corrected transposition of the great arteries. New England Journal of Medicine, 282, 1053-1059.

Lev, M Licata, R H, and May, R C (1963). The conduction system in mixed levocardia with ventricular inversion (corrected transposition). Circulation, 28, 232-238.

Rotem, C E, and Hultgren, H W (1965). Corrected transposition of the great vessels without associated defects. American Heart Journal, 70, 305-318.

Schiebler, G L, Edwards, J E, Burchell, H B, DuShane, J W, Ongley, P A, and Wood, E H (1961). Congenital corrected transposition of the great vessels; a study of 33 cases. Pediatrics, 27, 851-888.

Shem-Tov, A, Deutsch, V, Yahini, J H, Kraus, Y, and Neufeld, H N (1971). Corrected transposition of the great arteries. American Journal of Cardiology, 27, 99-113.

Requests for reprints to: Dr S Amikam, Department of Cardiology, Rambam Medical Centre, Bat-Galim, Haifa, Israel. 